\title{
G2677T polymorphism can predict treatment outcome of Malaysians with complex partial seizures being treated with Carbamazepine
}

\author{
S. Subenthiran ${ }^{1}$, N.R. Abdullah ${ }^{1}$, P.K. Muniandy ${ }^{1}$, J.P. Joseph ${ }^{2}$, \\ K.C. Cheong ${ }^{3}$, Z. Ismail ${ }^{1}$ and Z. Mohamed $^{4}$ \\ ${ }^{1}$ Bioassay Unit, Herbal Medicine Research Center, Institute for Medical Research, \\ Kuala Lumpur, Wilayah Persekutuan, Malaysia \\ ${ }^{2}$ Department of Neurology, Kuala Lumpur General Hospital, \\ Kuala Lumpur, Wilayah Persekutuan, Malaysia \\ ${ }^{3}$ Medical Research Center, Institute for Medical Research, \\ Kuala Lumpur, Wilayah Persekutuan, Malaysia \\ ${ }^{4}$ Department of Pharmacology, Faculty of Medicine, University of Malaya, \\ Kuala Lumpur, Wilayah Persekutuan, Malaysia \\ Corresponding author: S. Subenthiran \\ E-mail: soobitha@imr.gov.my
}

Genet. Mol. Res. 12 (4): 5937-5944 (2013)

Received September 24, 2012

Accepted March 26, 2013

Published November 26, 2013

DOI http://dx.doi.org/10.4238/2013.November.26.3

\begin{abstract}
Carbamazepine (CBZ) is used as the first line of treatment of complex partial seizures (CPS) in Malaysia. While this drug is known to be effective for the treatment of CPS, more than $30 \%$ of patients remain drug resistant to $\mathrm{CBZ}$ mono-therapy. We examined a possible relationship between patients' response to $\mathrm{CBZ}$ mono-therapy and the G2677T SNP of the ABCB1 gene. Three hundred and fourteen patients with CPS were recruited from the Neurology Department of the Kuala Lumpur Hospital, of whom 152 were responders and the other 162 were non-responders to CBZ mono-therapy. DNA was extracted from blood samples and real-time PCR was performed to detect the G2677T SNP of the ABCB1 gene. Results were described as genotype frequencies and
\end{abstract}


compared by logistic regression analysis. Among the 152 responders, $74 \%$ had the GG genotype. However, among the 162 non-responders, $26.5 \%$ had the GT genotype and $39 \%$ had the TT genotype. There was a significant difference in genotype frequency (TT vs GG; odds ratio $4.70 ; 95 \%$ confidence interval, 2.70-8.20) between responders and nonresponders. The presence of the T allele of the G2677T SNP appears to be a useful screening marker to determine if a patient is going to be resistant to CBZ as a single drug therapy in the treatment of CPS.

Key words: Complex partial seizure; Carbamazepine; Malaysians; ABCB1; G2677T

\section{INTRODUCTION}

Epilepsy is a complex of seizures that arise from abnormal, excessive, synchronous, and sustained discharge of a group of neurons. This in turn leads to a persistent increase in neuronal excitability. Half of all epilepsies are idiopathic, while other causes of epilepsy are trauma, oxygen deprivation, tumors, infection, metabolic derangement, neuronal migration disorders and monogenic epilepsies (Engelborghs et al., 2000). The incidence of epilepsy in Malaysia is 4-8 in 1000 (Kadir and Chadwick, 1999), while the percentage of complex partial seizures (CPS) of idiopathic etiology is 5.7\% (Pratap and Gururaj, 1989). Three mechanisms have been identified to be involved in the pathogenesis of epilepsy: excitability, interictal-ictal transition and neurochemical mechanism (Engelborghs et al., 2000).

\section{Carbamazepine}

Carbamazepine (CBZ) is used in the treatment of generalized tonic clonic, partial and generalized secondary seizures. CBZ is a derivative of iminostilbene, which is related to the tricyclic antidepressant family. This makes it suitable in the treatment of bipolar affective disorder and trigeminal neuralgia (Mayo Clinic, 2010).

In KLH, CBZ is used as the first line of treatment of CPS, since it is known to be used to treat seizures effectively in most individuals and also because it is economical. CBZ is transported bound to albumin and $\alpha 1$-acid glycoprotein (AGP), an acute phase protein. In normal individuals, $75-85 \%$ of the drug is bound to these plasma proteins, while $20-25 \%$ remains in the free form (Dasgupta, 2008). CBZ exhibits significant dose-response variability, so individualizing dosage and determining if a person is resistant to the drug is essential. Drug resistance is known to be influenced by failure in drug transport, which is thought to be governed by genetic factors (Ma and Lu, 2011). Single nucleotide polymorphism (SNP) in specific genes may be used to predict if a person will respond to a drug as well as the therapeutic dosage required for a successful therapy (Hunt, 2008).

\section{G2677T polymorphism (rs2032582)}

The G2677T SNP was the first SNP identified in the ABCB1 gene. It is also known 
as Ala893Thr and is located in exon 21. The ABCB1 gene is also known as the multi drugresistance 1 (MDR1) gene, and the gene product P-glycoprotein (P-gp) 170 is a member of the ATP-binding cassette transporter superfamily. ABCB1 is located on chromosome 7q21.1. The gene consists of 29 exons spanning nearly $200 \mathrm{~kb}$ of genomic DNA; however, only 27 exons code for P-gp. Its primary start site produces an mRNA transcript of $4360 \mathrm{bp}$ that contains 28 exons. P-glycoprotein has 1280 amino acids, 12 trans-membrane domains and two ATP-binding domains. ABCB1 is expressed in barrier and excretory tissues that have protective or elimination roles, such as the blood-brain barrier, intestines, liver, kidney and placenta (Riordan and Ling, 1985).

Pharmacotherapy of CNS disorders such as epilepsy, neurodegenerative disease and cerebral neoplasms are limited by the blood-brain barrier. P-gp is a critical element of the barrier (Miller et al., 2008). The factors that make P-gp a selective gate keeper of the blood-brain barrier are its high level of expression, luminal membrane location, multispecificity, and high transport capacity (Bauer et al., 2005). Almost a third of patients around the world are reported to have drug-resistant epilepsy, which is associated with an increased risk of death and debilitating psychosocial consequences (Wahab, 2010). As this form is resistant to multiple antiepileptic drugs (AEDs), the mode of resistance must involve drug efflux transporters such as ATP-binding cassette subfamily B member 1 (Bazil, 2003). The correlation between the presence of the T allele of the G2677T SNP and resistance to AEDs has been studied by scientists in different countries, such as Mosyagin et al. (2008) in Germany and Grover et al. (2010) and Vahab et al. (2009) in India, and was previously thought to be non-significant until patient response to an optimal dose of CBZ as a single drug therapy was studied in KLH.

\section{MATERIAL AND METHODS}

This study was approved by the Research Review Committee, Institute for Medical Research and Medical Research Ethics Committee (MREC), Ministry of Health Malaysia. The study was conducted in accordance with the principles stated in the Declaration of Helsinki.

\section{Recruitment of patients for study}

Malaysian patients of Malay, Chinese and Indian and East Malaysian ethnicity were recruited from the Epilepsy Clinic, Neurology Department of KLH, which is open on Monday afternoons. There were a total of 40 to 50 patients who were followed-up every Monday, since KLH serves as a tertiary referral hospital. The patient files were identified prior to the epilepsy clinic and written informed consent was obtained from suitable study volunteers. Study volunteers who fulfilled the inclusion criteria were enrolled into the study. They were provided with clear verbal as well as written information about the study. Those enrolled into the study gave their informed consent voluntarily.

A total of 314 patients were recruited and enrolled into the study. One hundred and fifty-six (49.7\%), 84 (26.8\%) and 70 (22.3\%), and 4 (1.2\%) were Malay, Chinese and Indian and East Malaysians, respectively. The study volunteers were diagnosed with CPS and secondary causes such as trauma, metabolic disorders, tumors and infectious causes were ruled 
out. As per the study design, 152 were responders to CBZ, while 162 were non-responders to CBZ. The study volunteers were required to answer a few questions regarding their family background (family history of epilepsy) their disease history (onset of the disease, characteristics as well of evolution of the seizures, frequency of attacks and treatment history) and progression (change in frequency of seizures after the onset of treatment) before they were assigned to the respective groups.

The responders included in the study comprised Malaysian citizens who were existing patients with CPS, under KLH Neurology Department follow-up. They were found to have normal MRI findings and mostly normal scalp EEG, except a few patients who showed left or right temporal spikes (Gupta and Jeavons, 1985). They were men and women between the ages 18 and 60 years with no other co-morbidity. They were already on an optimum dose of CBZ as a single drug therapy and were compliant with treatment. As they were being followed-up every 3 months, we had chosen patients with no episodes of seizures in more than a year. We made sure that their serum CBZ levels were within the therapeutic range (17-51 $\mu \mathrm{M})$ to ensure compliance with treatment.

The non-responders included in the study were Malaysian citizens who were existing patients with CPS with similar MRI and EEG findings as the responders, under KLH Neurology Department follow-up. They were men and women between the ages of 18 and 60 years with no other co-morbidity. They were on polytherapy that included CBZ, at the time of recruitment. They were treatment-naïve patients. Their compliance with treatment while they were on CBZ monotherapy was supported by their serum CBZ level before other antiepileptic agents were introduced.

Patients with other types of seizures were excluded from the study. Patients who were never treated with CBZ as well as those who had abnormal renal and liver function tests were also excluded from the study. In addition, patients who were on drugs that interacted with CBZ, such as alcohol, macrolides, antitubercular drugs, antifungal agents, benzodiazepines, antipsychotics, antidepressants, calcium channel blockers, cisplatin, H2 blockers, cyclosporine, doxorubicin, tetracyclines, hormone contraceptives, antiretroviral drugs and theophylline, were excluded from the study, to rule out confounding factors that could influence the outcome of treatment (Marks, 2008).

\section{Collection and processing of samples from the selected study volunteers}

A 5-mL blood sample was drawn from each study volunteer. Of this, $1 \mathrm{~mL}$ was transferred to 4-mL EDTA Vacutainer tube, while $2 \mathrm{~mL}$ were transferred to a 4-mL heparin Vacutainer tube and another $2 \mathrm{~mL}$ were transferred to a $4 \mathrm{~mL}$ plain Vaccutainer tube with gel. The blood in the plain tube with gel was for serum CBZ level, while the blood in the heparin tube was for liver function test and renal profile, unless the patient had recent documented results for these parameters. The blood in the EDTA tube was kept for DNA extraction. To avoid heme induced damage of the DNA, $3 \mathrm{~mL}$ G-Dex II RBC lysis buffer (Intron Biotechnology, USA) were added to the blood in the EDTA Vacutainer. The Vacutainers were gently inverted a few times. The samples were then taken to the Bioassay Laboratory at the Institute for Medical Research, where the EDTA Vacutainers were centrifuged at $2000 \mathrm{rpm}$ for $10 \mathrm{~min}$. The supernatant layer containing the lysed RBCs was then discarded while the lower layer was kept for DNA extraction. The samples for 
renal profile, liver function test and serum CBZ level were sent to the laboratory at the Kuala Lumpur Hospital to be processed. The results were later traced and reviewed.

\section{DNA extraction from whole blood}

DNA was extracted from the buffy coat using the Qiagene DNA extraction blood kit. DNA concentration and purity were determined using the Nanodrop 2000 Spectrophotometer (Thermo Scientific, USA).

\section{Real-time PCR}

Real-time PCR was run using SNP Specific Predesigned Taqman Probes (content sequence: TATTTAGTTTGACTCACCTTCCCAG[C/T]ACCTTCTAGTTCTTTCTTATCTT TC) as well as Vic and Fam reporter dyes. DNA samples were diluted to a concentration of $10 \mathrm{ng} / \mu \mathrm{L}$. The assays were run using a reaction volume of $10 \mu \mathrm{L}$ consisting of $5 \mu \mathrm{L}$ TaqMan GTXpress Master mix and $0.5 \mu \mathrm{L}$ TaqMan Drug Metabolism SNP Genotyping assay mix (Applied Biosystems, USA), $1 \mu \mathrm{L}$ diluted DNA, and 3.5 $\mu \mathrm{L}$ DNAse/RNAse free water (Germany). The reactions were run for 40 cycles at the cycling temperatures shown in Table 1 , for $40 \mathrm{~min}$.

\begin{tabular}{llcc}
\multicolumn{2}{c}{ Table 1. Step One Plus Real Time PCR setting. } \\
\hline Stage & Step & Temperature & Time \\
\hline Holding & DNA polymerase activation & $95^{\circ} \mathrm{C}$ & $20 \mathrm{~s}$ \\
Cycling $(40$ cycles $)$ & Denature & $95^{\circ} \mathrm{C}$ & $3 \mathrm{~s}$ \\
& Anneal/Extend & $60^{\circ} \mathrm{C}$ & $30 \mathrm{~s}$ \\
\hline
\end{tabular}

\section{RESULTS}

Among the 314 patients recruited, 156 were males while 158 were females, with a mean age of $37.6 \pm 12.3$ years. The responders were on a mean dose of $9.75 \pm 5.1 \mathrm{mg} \cdot \mathrm{kg}$ ${ }^{1} \cdot$ day $^{-1}$ and had a mean serum CBZ level of $27.5 \pm 10.7 \mu \mathrm{M}$.

The distribution of all genotypes (wild-type and heterozygous and homozygous mutant variants) and allelic frequencies are shown in Table 2. Genotype frequencies were in accordance with Hardy-Weinberg equilibrium. Multiple logistic regression analysis was as shown in Table 3.

Table 2. Frequency of the G2677T variant genotype in relation with patients' response to CBZ.

\begin{tabular}{|c|c|c|c|c|c|c|}
\hline & \multirow[b]{2}{*}{$\mathrm{N}$} & \multicolumn{3}{|c|}{ Genotype } & \multirow[t]{2}{*}{$\chi^{2}$ statistic $^{a}$ (d.f.) } & \multirow[t]{2}{*}{$P$ value } \\
\hline & & $\begin{array}{c}\text { GG } \\
\text { N (\%) }\end{array}$ & $\begin{array}{c}\text { GT } \\
\text { N }(\%)\end{array}$ & $\begin{array}{c}\text { TT } \\
\text { N }(\%)\end{array}$ & & \\
\hline Responders & 152 & $113(74.3)$ & $12(7.9)$ & $27(17.8)$ & $50.8(2)$ & $\mathrm{P}<0.001$ \\
\hline Non-responders & 162 & $56(34.6)$ & $43(26.5)$ & $63(38.9)$ & & \\
\hline All patients & 314 & $169(53.8)$ & $55(17.5)$ & $90(28.7)$ & & \\
\hline
\end{tabular}

${ }^{a}$ Chi-square for independence, Phi and Cramer's V $=0.402(\mathrm{P}<0.001)$. 
Table 3. Logistic regression analysis of the association between the responses to CBZ in the study population (N $=314$ ) on the basis of genotype at nucleotide position 2677 in exon 21 of the ABCB1 gene.

\begin{tabular}{lcccc}
\hline Genotype & Crude odds ratio & $95 \% \mathrm{CI}$ & Adjusted odds ratio $^{\mathrm{a}}$ & $95 \% \mathrm{CI}$ \\
\hline GG $v$ non-GG & $0.18^{*}$ & $(0.11,0.30)$ & $0.19^{*}$ & $(0.11,0.30)$ \\
GT vs non-GT & $4.23^{*}$ & $(2.13,8.36)$ & $4.10^{*}$ & $(2.05,8.19)$ \\
TT $v$ non-TT & $2.95^{*}$ & $(1.75,4.97)$ & $3.0^{*}$ & $(1.77,5.07)$ \\
TT $v$ GG & $4.71^{*}$ & $(2.71,8.19)$ & $4.70^{*}$ & $(2.70,8.20)$ \\
GT vs GG & $7.20^{*}$ & $(3.54,14.79)$ & $7.0^{*}$ & $(1.4 .5)$ \\
TT $v$ GT & 0.65 & $(0.30,1.42)$ & 0.67 & $(0.30,1.47)$ \\
\hline
\end{tabular}

$\overline{\mathrm{CI}}=$ confidence interval; $\mathrm{GG}=$ homozygous wild type; $\mathrm{GT}=$ heterozygous; $\mathrm{TT}=$ homozygous mutant; ${ }^{\text {adjusted }}$ for ethnicity and age. $* \mathrm{P}<0.001$.

\section{DISCUSSION}

A total of 314 patients with CPS were recruited from the Epilepsy Clinic at KLH, Malaysia between August 2009 and January 2011. They were recruited irrespective of race or gender. The percentage distribution of the patients based on race was reflective of our population. In addition, their renal profile and liver function test were determined to exclude the failure of therapy due to poor drug metabolism or elimination. A total of 152 patients were responders while the other 162 were non-responders to CBZ monotherapy. Patients were considered responders if they were free of unprovoked seizures for more than 12 months since the initiation of the optimum dose of CBZ as a single drug therapy. Non-responders were patients who had poor seizure control with optimum dose CBZ monotherapy, requiring the addition of another AED or the substitution with another drug all together. ABCB1 is a multidrug efflux pump known to be present in all excretory organs including the epithelial lining of the bloodbrain barrier. This P-gp macromolecule is responsible for drug transport. The high expression of the ABCB1 gene in many of these organs suggests that it is responsible for drug transport. Failure of drug transport could be associated with certain polymorphisms in the gene. SNPs associated with drug response were found to differ among different populations. The G2677T was the first SNP discovered for human P-gp. The association between the G2677T SNP in relation to the treatment outcome with CBZ among Europeans (Mosyagin et al., 2008) and Indians from India (Grover et al., 2010) were found to be non-significant. On the contrary, a similar study conducted in Japanese patients with temporal lobe epilepsy (Seo et al., 2006) found a significant association. A study carried out in the Chinese population of Beijing showed a high degree of linkage disequilibrium between G2677T and two other polymorphisms of the ABCB1 gene in relation to drug-resistant epilepsy (Lu et al., 2007). However, when the G2677T SNP was examined in a single locus analysis in the same study, it failed to show significant association with drug-resistant epilepsy. An important factor that was not considered was the type of seizure and the etiology of seizures among the patients recruited in the studies.

A previous study conducted in Malaysia showed that there was no association between polymorphisms of the ABCB1 gene and drug-resistant epilepsy. However, it did not specify the type of epilepsy or mentioned the specific drug chosen to be studied. Drug interaction between AEDs and other drugs were not ruled out. (Haerian et al., 2011). The team that conducted the study in the Japanese population only recruited patients with temporal lobe seizures, which are responsible for most CPS and managed to obtain a significant association between the G2677T polymorphism and the outcome of treatment. Prompted by the Japanese 
study, we decided to focus on CBZ monotherapy as the first line of treatment for CPS, since it is practiced in KLH. Another important confounding factor we eliminated was known drug interaction with CBZ. Some of the patients, who we had to exclude from the study initially responded well to CBZ monotherapy until a calcium channel blocker such as amlodipine and nifedipine was introduced for newly diagnosed hypertension in these patients. Patients who did not respond to CBZ monotherapy but were found to be on concurrent treatment with theophylline for the treatment of bronchial asthma were also excluded for the same reason. We found that patients who had the GG genotype were significantly $(\mathrm{P}<0.001)$ more likely to respond to CBZ monotherapy ( $74.3 \%$ of the responders). Among the responders, $7.9 \%$ had the GT while another $17.8 \%$ had the TT genotype. However, among the non-responders, $38.9 \%$ had the TT genotype, $26.5 \%$ had the GT genotype and $34.6 \%$ had the GG genotype. On the basis of logistic regression analysis, a patient with the GG genotype was significantly $(\mathrm{P}<$ 0.001 ) more likely to respond to an optimal dose of CBZ as compared to those who were heterozygous or had the TT genotype. However, a patient with the TT genotype was more likely to be resistant to $\mathrm{CBZ}$ as compared to patients with the GG and GT genotypes. The majority of the non-responders carried the $\mathrm{T}$ allele $(66.2 \%)$. This shows that the $\mathrm{T}$ haplotype may be responsible for drug resistance to $\mathrm{CBZ}$ in patients with complex partial seizures. As for the $34.6 \%$ of the non-responders who had the GG genotype, factors such as stress and sleep deprivation leading to drug-refractory epilepsy were probable but could not be determined. Neither the drug response nor the genotype showed any correlation with race.

All 152 patients who were responders to CBZ monotherapy achieved good seizure control and a significant improvement in their quality of life. In fact, quite a few of them were in the process of being weaned off CBZ after being seizure-free for almost 3 years. The results suggest that screening patients for the T haplotype of the G2677T SNP maybe a reliable way to predict drug resistance among patients with complex partial seizures before initiating CBZ therapy.

\section{ACKNOWLEDGMENTS}

Research supported by the National Institute of Health, Ministry of Health, Malaysia. We thank the Director General of Health, Ministry of Health, Malaysia and Director of the Institute for Medical Research, Dr. Shahnaz Murad, for giving us the permission to publish this paper. We are grateful to the Pharmacology Department, Faculty of Medicine, University of Malaya for training and assistance. We also thank the Head of Department, Dr. Md Hanip Rafia and the staff of the Neurology Department, Kuala Lumpur General Hospital for allowing us to use their facilities to recruit patients for the study.

\section{Conflicts of Interest}

The research was conducted in the absence of any commercial or financial relationships that could be construed as a potential conflict of interest.

\section{REFERENCES}

Bauer B, Hartz AM, Fricker G and Miller DS (2005). Modulation of p-glycoprotein transport function at the blood-brain barrier. Exp. Biol. Med. 230: 118-127. 
Bazil C (2003). Drug resistant epilepsy: A compliance problem or an abnormality of transport proteins? Epilepsy Curr. 3: 204-206.

Dasgupta A (2008). Handbook of Drug Monitoring Methods: Therapeutics and Drugs of Abuse. In: Therapeutic Drug Monitoring: Therapeutic Drug Monitoring of Anticonvulsants (Dasgupta A, ed.). Humana Press, New Jersey, 23.

Engelborghs S, D’Hooge R and De Deyn PP (2000). Pathophysiology of epilepsy. Acta Neurol. Belg. 100: 201-213.

Grover S, Bala K, Sharma S, Gourie-Devi M, et al. (2010). Absence of a general association between ABCB1 genetic variants and response to antiepileptic drugs in epilepsy patients. Biochimie 92: 1207-1212.

Gupta AK and Jeavons PM (1985). Complex partial seizures: EEG foci and response to carbamazepine and sodium valproate. J. Neurol. Neurosurg. Psychiatr. 48: 1010-1014.

Haerian BS, Lim KS, Mohamed EH, Tan HJ, et al. (2011). Lack of association of ABCB1 and PXR polymorphisms with response to treatment in epilepsy. Seizure 20: 387-394.

Hunt S (2008). Controversies in Treatment Approaches: Gene Therapy, IVF, Stem Cells, and Pharmacogenomics. Nature Edu. 1. Available at [http://www.nature.com/scitable/topicpage/controversies-in-treatment-approaches-genetherapy-ivf-792\#].

Kadir ZA and Chadwick DW (1999). Principles of treatment of epilepsy. Drugs Today 35: 35-41.

$\mathrm{Lu}$ Q, Wu L, Jin L, Xu Q, et al. (2007). Association analysis of a polymorphism of MDR1 gene and refractory temporal lobe epilepsy in a Chinese population. Neurol. Asia 12: 94-95.

Ma Q and Lu AY (2011). Pharmacogenetics, pharmacogenomics, and individualized medicine. Pharmacol. Rev. 63: 437-459.

Marks JW (2008). Carbamazepine, Tegretol, Tegretol XR, Equetro, Carbatrol. Available at [http://www.medicinenet.com/ carbamazepine/article.htm]. Accessed January 23, 2008.

Mayo Clinic (2010). Anti-Seizure Medications: Relief from Nerve Pain. Available at [http://www.mayoclinic.com/health/ pain-medications/PN00045]. Accessed November 18, 2010.

Miller DS, Bauer B and Hartz AM (2008). Modulation of P-glycoprotein at the blood-brain barrier: opportunities to improve central nervous system pharmacotherapy. Pharmacol. Rev. 60: 196-209.

Mosyagin I, Runge U, Schroeder HW, Dazert E, et al. (2008). Association of ABCB1 genetic variants 3435C $>\mathrm{T}$ and $2677 \mathrm{G}>\mathrm{T}$ to $\mathrm{ABCB} 1 \mathrm{mRNA}$ and protein expression in brain tissue from refractory epilepsy patients. Epilepsia 49 : 1555-1561.

Pratap RC and Gururaj AK (1989). Clinical and electroencephalographic features of complex partial seizures in infants. Acta Neurol. Scand. 79: 123-127.

Riordan JR and Ling V (1985). Genetic and biochemical characterization of multidrug resistance. Pharmacol. Ther. 28: 51-75.

Seo T, Ishitsu T, Ueda N, Nakada N, et al. (2006). ABCB1 polymorphisms influence the response to antiepileptic drugs in Japanese epilepsy patients. Pharmacogenomics 7: 551-561.

Vahab SA, Sen S, Ravindran N, Mony S, et al. (2009). Analysis of genotype and haplotype effects of ABCB1 (MDR1) polymorphisms in the risk of medically refractory epilepsy in an Indian population. Drug Metab. Pharmacokinet. 24: $255-260$

Wahab A (2010). Difficulties in treatment and management of epilepsy and challenges in new drug development. Pharmaceuticals 3: 2090-2110. 